\title{
MARKET FLUCTUATIONS EXPLAINED BY DIVIDENDS AND INVESTOR NETWORKS
}

\author{
MATTHEW OLDHAM \\ Department of Computational and Data Sciences, \\ 4400 University Drive, Fairfax, Virginia 22030, USA \\ moldham@gmu.edu
}

Received 23 November 2016

Revised 15 June 2017

Accepted 3 August 2017

Published 29 August 2017

\begin{abstract}
The inability of investors and academics to consistently predict, and understand the behavior of financial markets has forced the search for alternative analytical frameworks. Analyzing financial markets as complex systems is a framework that has demonstrated great promises, with the use of agent-based models $(\mathrm{ABMs})$ and the inclusion of network science playing an important role in increasing the relevance of the framework. Using an artificial stock market created via an ABM, this paper provides a significant insight into the mechanisms that drive the returns in financial markets, including periods of elevated prices and excess volatility. The paper demonstrates that the network topology that investors form and the dividend policy of firms significantly affect the behavior of the market. However, if investors have a bias to following their neighbors then the topology becomes redundant. By successfully addressing these issues this paper helps refine and shape a variety of additional research tasks for the use of ABMs in uncovering the dynamics of financial markets.
\end{abstract}

Keywords: Agent-based model; artificial stock market; networks; dividend policy.

\section{Introduction}

The Efficient Market Hypothesis (EMH) [8] underlies much of today's mainstream finance theory and practices, a point reaffirmed by Summers [38] when he stated that the assumption of market efficiency "forms the basis for most research in financial economics". Despite some empirical support for the EMH, and the models that utilize its assumptions, the reality of continued episodes of extreme volatility, booms, and crashes has led some to consider alternatives. The search for alternatives has gained further impetus following the upheaval in global markets in 2007, which plunged the world into the Global Financial Crisis (GFC) that, according to the IMF, cost the global economy USD 11.9 trillion [6]. One theory that utilizes the EMH

This is an Open Access article published by World Scientific Publishing Company. It is distributed under the terms of the Creative Commons Attribution 4.0 (CC-BY) License. Further distribution of this work is permitted, provided the original work is properly cited. 
is the capital irrelevance theory [27], where it was initially proposed that the capital structure, and therefore the dividend policy of a company was irrelevant in determining a company's value, a claim tested in this paper.

To gain an understanding of why financial markets fail to comply to the prescribed behavior, at least in terms of the EMH, the use of a complex systems framework has become increasingly popular and relevant. For a system to be considered complex, it must contain some, if not all, of the following: feedback, nonstationarity, many interacting agents, adaption, evolution, single realization, and be open [15]. This position is consistent with the views of Sornette [37], who concluded that to understand stock market returns, one must consider: imitation, herding, selforganized co-operativity, and positive feedbacks. If one is to accept that financial markets operate as a complex system, then one must accept that the behavior of the system is an emergent process based on the self-organized behavior of independently acting, self-motivated individuals [10].

An additional benefit of utilizing a complex systems framework is that it allows networks to be included, something that has become an important consideration with the growth of network science and the availability of data. The case for the increasing utilization of network science within a complex system view of the economy comes from the fact that due to the increasing dependency between economic agents, it is becoming increasingly difficult to predict and control the economy [35]. In a more specific application of networks to financial markets, they have been able to explain trading decisions and portfolio performance [30] and networks have been found to exist between investors [36].

The use of agent-based models (ABMs) has been become a primary tool in trying to understand the dynamics of a complex system, and a large volume of work utilizing ABMs to create artificial stock markets has been developed (see [19, 37] for extensive reviews of the application of ABMs to financial markets). The key rationale for the use of ABMs is that they are not constrained to equilibrium conditions [37], with the utilization of network structures between agents within these models providing a fertile stream of research.

This paper implements an artificial stock market ABM that not only makes use of differing investor networks but also varies the dividend payout ratio of the risky asset traded in that market, with the intent of understanding under what conditions, if any, that the capital irrelevance theory accurately reflects the outcome recorded by the model. The various experiments employed to meet the research objective produces multiple considerations for management when implementing a dividend policy, and for investors in general. These include the topology of the network their investors are linked through, the need to appreciate what is driving the share price of a company at any particular time, momentum or fundamentals, and how investors are assessing the various sources of information available to them.

The remainder of this paper is structured in the following manner: the background to and the support for this paper (Sec. 2), followed by a review of the model underlying this paper, including both the original model of Harras and Sornette [13] 
and the author's extensions (Sec. 2). The extensions cover the use of differing network topologies (Sec. 3.2) and the introduction of dividends as determined by a userdetermined payout ratio (Sec. 3.1). The results of the various experiments are then presented with the results from the different experimental settings compared and contrasted (Sec. 4). Conclusion and discussion (Sec. 5) are provided to review the significance of the findings and highlight future research opportunities.

\section{Background}

While the origins of the EMH and the principle that stock prices are unpredictable can be traced back to the early 1900's [3], it was not until the 1950's that it gained traction in the broader economic community (see [24, 40] for a comprehensive account of the development of the EMH and its application in modern finance). For the EMH, efficiency refers to the fact that all information is rapidly reflected in the price and there is no information capable of moving the share price that is not already incorporated in the price. In turn, because news arrives in a random unpredictable fashion, prices will follow a similar pattern, resulting in returns and the asset's price following a random walk. Further, returns are assumed to follow a Gaussian distribution, noting that it was originally suggested that prices would follow such a distribution [3]; however, this was revised when it was proposed that it was returns [28], rather than prices that followed a Gaussian distribution [40]. Implicit within this assumption is the fact that periods of extreme price volatility, asset bubbles, and subsequent crashes, will not and cannot occur.

Due to there being no precise definition for a financial bubble, nor consensus on how to detect them, or the fundamentals that are responsible for creating them there has much debate on whether they exist [41]. A straightforward definition of a bubble is a boom that ended with a crash, with a boom being defined as a doubling of a market's value within a year, and a crash a halving of its value within the same time-period [11]. Periods of high price to earnings (PE ratio) is an alternate metric associated with markets potentially being in a bubble [11], because it represents a period where the assets' prices are not supported by their fundamental earnings. However, not all periods of excessive asset valuations equate to a bubble because a crash, the steep decline in prices, that is required to classify the period of high PE's as a bubble, does not eventuate.

An alternative view to how financial markets have performed is that a Gaussian model provides a rough approximation of financial market returns, but fails to explain outlying events [17]. One such outlying event was the crash that occurred in global markets in the October of 1987, when the Dow Jones index fell $22.6 \%$ in a day. Under the assumptions underlying the random walk theory, the probability of such an event is 1 in 50 billion [26]. It is the underestimation of such an event that has driven the search for alternative theories to explain the outlying events.

A further view of the return characteristics of financial markets, as summarized by $[5,15]$, is that they demonstrate: excess volatility - the existence of large 
movements not supported by the arrival of new news; heavy tails — returns exhibit "heavy-tail" or "fat-tail" indicating that returns deviate more than anticipated and do not follow a Gaussian distribution; volatility clustering - large changes are followed by further large changes; and volume/volatility clustering — trading volumes and volatility show the same type of long memory. In addition, Mandelbrot [25] first provided evidence that returns may follow a unique distribution, a powerlaw distribution, see [22] for a detailed review of the empirical evidence supporting the existence of power laws in financial markets. For investors, the main implication of returns following a power-law is that the risk of large losses is much higher than suggested by the EMH, and markets are more volatile. It is the existence of powerlaw returns that provides the key insight that financial markets may operate as a complex system.

To gain an understanding of the dynamics of a complex system, ABMs have become a key tool as they allow for interaction between individual agents (investors, in the case of financial markets) who act and undertake actions based on the context of their environment while utilizing a basic set of rules. The agents' behavior is not fixed and can evolve in response to the behavior of other agents and their environment. Another advantage is that ABMs are not constrained to equilibrium conditions [37], which is the key assumption underlying traditional neo-classical models of finance and economics in general. ABMs also provide researchers with the option of creating niche focused models to explore a specific issue, such as the model presented in this paper, or a holistic economy wide model intended to explore the implications of policy intervention, as demonstrated by the EURACE project [7]. Regardless of the modeling approach the focus of the research with regards to artificial stock markets has been to reproduce the stylized facts of financial markets, and identifying the conditions under which the return characteristics match the EMH or models that utilize it.

Since the original ABM-based artificial stock market (see [2]) there have been numerous approaches employed to uncover the true dynamics of financial markets. The various frameworks can be divided into four categories [5]: heterogeneous arrival of information; evolutionary models; behavioral switching; and investor inertia. The differentiating factors for each framework is how they handle agent preferences, the pricesetting mechanism, whether evolution is allowed, and how strategies are stored. Despite the differences, each framework has the common theme of utilizing heterogeneous agents in terms of both expectations and investment strategies with the intention of: studying how the agents act and prices change; reproducing the stylized facts of the markets, and most importantly understanding the influence of the market's microstructure. The models are not without issues, and in particular the early models as they tended to be complex, making it difficult to determine the influence of each of the inputs and what the key determining factors were [20]. Further, the predictive power of a given model can be constrained and it is unclear how to generalize a given result [37].

The inclusion of networks within the various ABM-based artificial stock markets is now a growing field of research. The belated use of this technique most likely 
resulted from the fact that the work on artificial stock markets predated the meteoric rise in the applications of network theory in the 2000s. Additionally, given the level of computing power available at the time and the computational intensity required, the inclusion of networks was not feasible. The benefits of adding networks have since been demonstrated when, the real-world market returns were replicated in a model that utilized social interaction amongst investors [14]; the network structure of investors was shown to influence the stability of, and the fluctuations of, an asset's price [32]; and, it was demonstrated how bubbles can emerge because of agents considering different information sources, including the expected actions of their neighbors [13].

Given the importance of dividends to the total return of a financial asset (for example, for the S\&P 500, dividends are responsible for $42 \%$ of total returns [34]), it is important to gain a greater understanding into the possible affect they have in the decision-making process of the investors. Regardless of the role dividends play in the decision-making process of the investor, it should be noted that the reason why firms pay a dividend is an area of ongoing discussion, with no decisive evidence supporting the argument that they are used to signal favorable information to the market or to mitigate agency problems [21]. An alternative view has been provided by firstly [4] and supported by, [21], with the proposed dividend catering theory. Under the theory, investors' demand for higher yield stocks, and therefore any premium that the dividend paying firms attract, is dependent on the investors' appetite for dividends at any particular time, which in turn varies based on the market conditions [21]. The model presented in this paper complements this theory by presenting, and explaining, the dynamics of how the demand for yield is capable of driving the prices of assets above what may be considered their fundamental level.

One interpretation of how investors should consider dividends is the capitalstructure irrelevance theory [27]. The underlying principle is that the market value of a company comes from its earning power and the risk of its underlying assets, therefore making its value independent of the way it finances investments or distributes dividends. A further consideration is that if a company has high growth prospects, its market value should be higher and hence its stock prices would be high. In turn, if a company is lacking attractive growth prospects, the market value of that company will be lower. The EMH is one of the key assumptions used to justify this proposition, because for the theory to hold, all investors must have access to all relevant information and the information is fully reflected in the price of the asset. The implication of these points being that if the theories hold true then the introduction of a dividend should have no effect on the risky-asset's price, a point tested in this paper.

The focus of this paper is to test the validity of the proposed behavior of the investors under capital-structure irrelevance via an ABM-based artificial stock that has investors linked via varying network topologies. As such, the paper takes the important step of fusing together the works of ABM-based artificial stock markets and corporate finance literatures. The relevance being that while the use of dividends 
to invoke varying investor behavior is quite common in ABM-based artificial stock markets, the existing literature has tended to treat the dividend as an exogenous variable generated through a stochastic process. In contrast, the current model combines a stochastic earnings per share (EPS) variable, investor expectations through an endogenous consensus forecasting process, and a user-determined dividend payout ratio for the risky asset. The approach is justified as this dividend payment process more closely resembles what occurs in the corporate world, thus opening a new line of research, which is to understand if and how management can manipulate its stock price using its dividend policy, given the underlying earnings' potential of the company remaining comparable. This topic is particularly relevant given the low central bank interest rates, and the subsequent search for alternative yield by investors at the time of the research.

The model is also capable of analyzing the effect the alternative investor network structures have on the performance of financial markets, with a focus on understanding whether certain network structures lead to greater volatility, and analyzing the dynamics behind whether or not the population forms large common groups ("herds") in terms of their investment strategies. Therefore, the causation of several documented effects of the investor networks, including, how the topology of a social network affects information efficiency [30], and the role the centrality plays in determining the dynamics of the market $[29,31]$ can be investigated. The relevance of understanding the dynamics behind herd formation is that it provides insight into how bubbles can form in financial markets.

\section{Methodology}

From the wide array of model frameworks that exist, the artificial stock market as detailed by Harras and Sornette [13] was used as the foundation for this paper. Various extensions were then implemented to address the specific research questions, with the final model implemented in NetLogo 5.3. The justification for utilizing the aforementioned model is that it was able to generate price movements that were affected by investors who were influenced in their decision-making process by their neighbors, and the asset returns matched the stylized facts of financial markets that yet did not match the Gaussian distribution of the public and private information. In addition, the model can generate a significant bubble as investors firstly join a buying herd, before switching to the selling herd, as the returns of the risky-asset no longer justified an investment in it. The occurrence of the bubble is the result of a positive feedback loop, with regard to investors adopting the actions of their neighbors, becoming the dominate influence in the decision-making process of the investors [13]. However, the influence of the positive feedback is conditional on the initial bias that investors have to trust the actions of their neighbors.

To maintain the focus of the paper on the results rather than the minutiae of the model, a brief description of the original model and the author's extensions follows. A more detailed description of the workings of the model via a design and 
details document (ODD), along with the source code, is available at www.openabm. org/model $/ 5203 /$. To replicate the results of this paper, when using the model, the user should set the switches for the $H \& S$ and eff_frontier parameters to off, these are found under the Output Options heading. Further settings relevant to this paper are detailed in the results section (see Sec. 4).

The model is initialized with two classes: the risky asset and the investors. In turn, when the investors are initialized they are connected in a network as determined by the user, as discussed in Sec. 3.2. The key variables the asset owns are its payout ratio, its price (initiated at 1), and the mean and standard deviation of its EPS, the relevance of which is discussed in Sec. 3.1. The key investor variables include: the influence of the various information sources, their transaction ratio $(t r)$, and the transaction threshold. The transaction threshold is heterogeneous across the population and is drawn from a uniform distribution between 0 and the user-defined value at initiation. Separately, the market depth $(\lambda)$, which is used in the calculation of the new asset price, is set at initiation. In terms of the repeated steps, Fig. 1. provides an illustration of how the model operates at each step.

The basic premise behind the original model is that the boundedly rational investors have access to three sources of information (the expected actions of their neighbors $\left(E_{i j}\left[a_{i k}(t)\right]\right)$, public information $\left(p i_{i}(t)\right)$, and private information $\left(\epsilon_{i j}(t)\right)$ that they utilize to determine their propensity to invest $\left(\omega_{i j}\right)$ in a risky asset as per Eq. (1). In terms of notation, $i$ refers to the $i$ th asset, $j$ the $j$ th investor, $k$ the $k$ th neighbor of agent $j$, and $K$ being the number of neighbors agent $i$ has. To determine the expected actions of their neighbors, each investor polls their neighbors' actions to see if they are buying, selling, or holding their risky assets at each step. The other information sources are generated by a random draw from a normally distributed

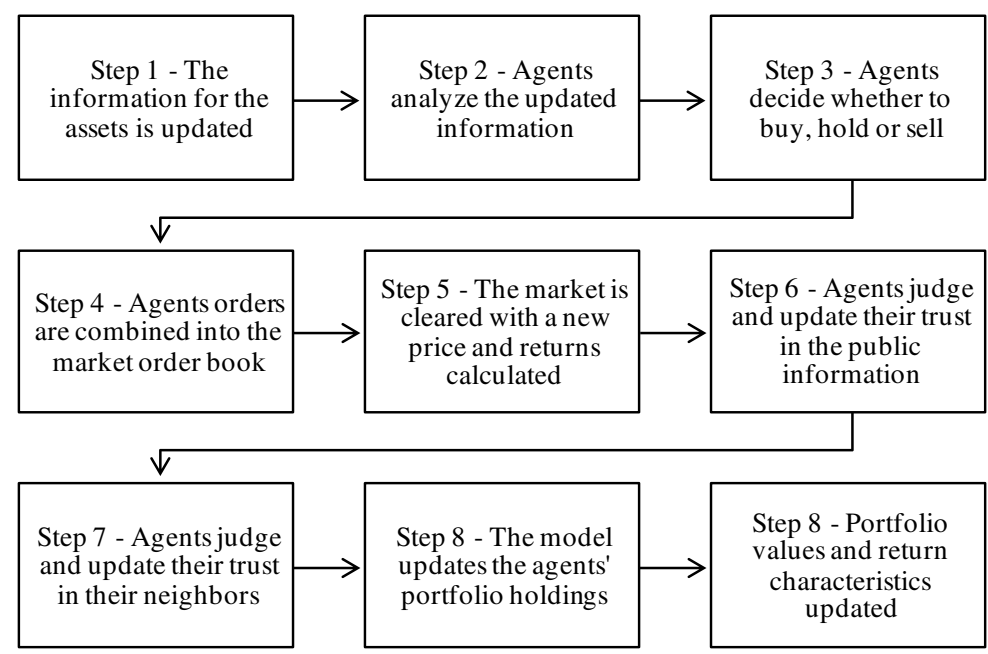

Fig. 1. Flow diagram of the repeated steps in the implemented model. 
probability function. The investor then compares their transaction thresholds to their resulting $\left(\omega_{i j}\right)$ to determine their actions.

$$
\omega_{i j}=c_{1 i j}\left(\sum_{k=1}^{K} n t_{j k}(t-1) E_{i j}\left[a_{i k}(t)\right]\right)+c_{2 i j} p t_{i}(t-1) p i_{i}(t)+c_{2 i j} \epsilon_{i j}(t) .
$$

The level of influence of each information source is weighted by a combination of up to two variables. For the $c_{1 i j}, c_{2 i j}$, and $c_{3 i j}$ variables, investors are initiated with a fixed value that is drawn randomly from a uniform distribution between 0 and a userdefined value. With the variable being used to weight how the investor assesses the relevant information source, an acceptable interpretation is that a higher value (such as 4) indicates a higher initial bias towards the relevant information source. As investors have a different value for these variables a level of heterogeneity is introduced within the population. It is by altering the $c_{1 i j}, c_{2 i j}$, and $c_{3 i j}$ coefficients that different dynamics are generated. In particular, when the upper limit for $c_{1 i j}$ is set at 4 , bubbles in the risky asset's price appear. Hence, analyzing the effect of different levels for this variable and $c_{2 i j}$ forms a key component of this paper and the original paper [13].

An important consideration in designing an ABM-based artificial stock market is the mechanism under which the price is determined. Options include establishing order books and fulfilling those orders through an auction process, or making use of a market-maker who co-ordinates the market. The early models tended to favor the market-maker approach while the later models have turned to a formal auction market. In what some may consider "hand waving", the market-maker can clear the market by providing liquidity and standing on the other side of all trades at each tick. This means that investors are guaranteed to have their trades executed and allow for price determination to occur at each step. Therefore, the market will not become frozen due to a lack of liquidity or an inability to match orders, something that is a real-world consideration. In addition, returns are not affected by large gaps in a discrete order book. The downside to this approach is that the model loses the ability to assess what the investors were willing to pay and accept for the asset. In the model utilized in this paper, the price of the risky-asset is endogenously determined via Eq. (4) (see Sec. 3.2 for an explanation) after each investor makes their investment decisions, and submits their buy or sell orders (step 5 in Fig. 1).

Investors then use the subsequent asset returns to reassess and adjust the trust they have in each of their information sources via the network trust $\left(n t_{j k}\right)$ and public trust $\left(p t_{i}\right)$ coefficients (steps 6 and 7 in Fig. 1). These variables are initiated at 0 , with the investors' trust based on the ability of the information source to predict an appropriate action, an appropriate action being when the information tells the investor to buy and the price subsequently increases (and vice-versa for a sell signal). 


\subsection{Model extension — Dividends and earning expectations}

To remove a level of abstraction in the original model a new source of public information and dividend process were introduced. The first change was to include an EPS value for the risky asset on the basis that it reflects the income-generating ability of the asset and is a key component in determining the fundamental value of an asset. The EPS value at each step is drawn from a Gaussian distribution, in a similar manner as the original model, but with the probability density function (PDF) having a mean equal to the initial price of the asset divided by the model's time-frame which is then further divided by an appropriate price earnings (PE) ratio. The extended model uses a quarterly time-frame with an initial price and PE of 1 and 15. The standard deviation of the EPS is set by the user, and for the results of this paper it was set at $50 \%$ of the mean. Based on this, the fundamental value of the risky-asset remains at 1 throughout the life of the model, with any period of sustained movement away from this price giving rise to a potential bubble.

A consensus earnings forecast is also included as in a given financial bubble, it is the expectation of future earnings rather than present economic reality that motivates the average investor [37]. The model computes the forecast by using a moving average of the asset's EPS history with an exponentially decreasing kernel, as determined by the memory_weight_parameter $(\alpha)$. The significance of the parameter is that it sets the time scale over which past performance affects a variable's value, which is given by $(\mid 1 / \ln ($ memory_weight_parameter $) \mid)$.

To determine the value of public information, the extended model has the investors assess the actual EPS for the asset at each tick against the consensus forecast, as per Eq. (2). If the actual earnings exceed the consensus estimate $\left(p i_{t i}>0\right)$, this is considered an earnings' surprise, resulting in a buy signal, and viceversa for a miss. If earnings meet expectations, then the information adds no value because the investor assumes the information is already reflected in the price. Support for the extension comes from the volume of work that shows stock prices that react positively to positive earnings' news, yet it takes time for this information to be reflected in the price of the asset [18].

$$
p i_{i}(t)=\frac{\operatorname{eps}_{i}(t)-\operatorname{eps} f_{i}(t)}{\operatorname{eps} f_{i}(t)} .
$$

In a further extension, the risky asset returns a dividend [DPS as defined by $d_{i}(t)$ ] if the EPS for a period is greater than 0 . The extended model determines the assets' dividend as per Eq. (3). The justification for the use of a payout ratio, as opposed to an absolute dividend is that a firms payout ratio is dependent on a combination of various accounting metrics including profitability, cash flow, and debt to equity ratio [11]. The payout ratio is set by the user at initiation.

$$
d_{i}(t)=\operatorname{eps}_{i}(t) * \text { pay_out_ratio }{ }_{i}
$$


Equation (4) provides an explanation of how the inclusion of a dividend affects the return of the asset in any given time period $\left(r_{i}(t)\right)$. The first half of Eq. (4) $\left(\frac{1}{\lambda * J} \sum_{j=1}^{N} a_{i j}(t) * v_{i j}(t)\right)$ deals with how an excess or deficiency in demand, as given by $\sum_{j=1}^{N} a_{i j}(t)$, for the asset affects its price when the market clears. The role of the $\lambda$ term is to weigh the investors' actions by the market depth, where $\lambda$ is defined as liquidity and $J$ is the total number of investors. The justification for the approach is that while it is only an approximation compared to the use of an actual order book, it provides a reasonable result [9]. The greatest downside is that the process assumes a permanent market effect between the clearing processes.

The second half of Eq. (4) $\left(\log \left(\left(d_{i}(t)+p_{i}(t)\right) / p_{i}(t)\right)\right.$ is the mechanism by which the inclusion of the dividend affects the return. Unsurprisingly, the presence of the dividend will boost the returns for investors. Therefore, when investors reassess their trust based on the returns of the asset if the price increased, the level by which the trust is reassessed is amplified upwards due to the dividend. However, if the price declines after a negative news, the signal is suppressed due to the dividend (on the condition that the EPS was $>0$ ). Therefore, investors will tend to revise upwards their trust at a faster rate than they revise it downwards. The ramifications of this are assessed in Sec. 4. Despite receiving a dividend, investors do not have access to those funds for reinvesting. This ensures that the extended model remained consistent with the original model, which was closed to new funds.

$$
r_{i}(t)=\frac{1}{\lambda * J} \sum_{j=1}^{N} a_{i j}(t) * v_{i j}(t)+\log \left(\left(d_{i}(t)+p_{i}(t)\right) / p_{i}(t)\right) .
$$

The relevance of Eq. (4) to the dynamics of the model is shown through Eq. (5) (noting that this equation relates solely to a single risky asset implementation of the model). The equation relates to Step 7 in Fig. 1, and shows how the agents update their trust in each of their neighbors $\left(n t_{j k}(t)\right)$ in each period. A similar equation is also used for the updating of the trust the agents have in their public information, and this is updated in Step 6 of Fig. 1.

$$
n t_{j k}(t)=\alpha * n t_{j k}(t-1)+(1-\alpha) E_{i j}\left[a_{i k}(t-1)\right] * \frac{r_{i}(t)}{\sigma_{i r}(t)} .
$$

The first part of the above equation discounts the previous value of the trust by the variable $(\alpha)$, which is set by the memory_weight_parameter. The significance of the $\alpha$ variable (the default setting in the model is 0.95 , which equates to a memory of 20 periods), is that it dictates the length of time that the value of the the "scaling term" at time $t$ continues to affect the level of the trust variable. The role of the "scaling term" $\left(\frac{r_{i}(t)}{\sigma_{i r}(t)}\right)$ is to normalize the past return of the asset by the standard deviation of its past returns. The rationale is that a larger return scaled by its volatility $\left(\sigma_{i r}(t)\right)$ will enhance the trust to a greater degree[13]. It should be clear to the reader that the inclusion of the dividend, as seen in Eq. (4), will inflate the "scaling term" resulting in an accelerated increase in either of the trust variables 
when they have provided correct advice in the past. This is a very important point because it should amplify the speed at which investors will increase the trust they have in an information source. Therefore, the speed at which the population herds, resulting in a bubble or crash, will increase.

\subsection{Model extensions - Networks}

The rationale for utilizing networks is that across a variety of research fields it has been proven that the behavior of a system can vary greatly depending on the network topology by which its agents are connected. However, while actual investor networks and their behaviors have been successfully uncovered [26], or implied through data analysis [31], it is an extremely difficult task to do so, thus providing the justifications for utilizing simulations, such as the one as provided in this paper, to investigate the possible underlying relationship between the investor networks and the behavior of financial markets. By using simulations, the claims about how the social network of the investors affects the information efficiency of markets [30] and how the resulting centrality characteristic affects the dynamics of a financial market [29, 31], can be more thoroughly examined. These works provide the motivation and reference points for the network extensions made to the implemented model, that is the model is designed to assess the effect on the pricing behavior of a risky asset resulting from changing the topology of the network that connects the investor population.

Within the network science literature there are four general types of network: regular/lattice, random/Erdos-Renyi, small-world, and scale-free, all of which are initially assessed in this paper. In high-level terms, the differences relate to how each agent is assigned neighbors and the number of neighbors they have. The differences are evident through various network specific metrics such as: degree distribution (the number of neighbors an agent has), centrality (where an agent is located in the network), and clustering coefficient (how the agents are grouped), and the diameter of the network (the steps required to reach other agents). It is the differences in these variables that are likely to be contributors as to why the pricing behavior may change for the different topologies. The model used in this paper has the flexibility to consider these networks, with a range of settings including: the number of neighbors an investor has and the probability that the investors connect to each other.

A lattice network has agents joined to a given number of agents that immediately surround them. This means that lattice networks are characterized by their agents being highly clustered but within the diameter of the network, that is the maximum number of steps required for any agent to reach another agent being high. In addition, each agent has the same number of neighbors, that is the degree distribution is uniform. While lattice networks are not common in the real world, they may be relevant to financial markets given the location of investors on trading desks. Despite its abstract nature, a lattice network provides a meaningful baseline by which the 
researchers can investigate the dynamics of a system, and in particular how the agents' behaviors change as a given shock diffuses through the population.

A small-world network is more consistent with the social networks of people, and is premised on the fact that the geographical proximity plays an important role in the formation of social networks. Agents are mainly linked to those agents immediately around them but importantly they are linked to a small number of long-distance neighbors. This distinction from the lattice network sees the small-world network maintain a high degree of clustering but due to the long-distance connections the overall diameter of the network is much smaller, meaning that in general it takes less number of steps for a given agent to reach another agent within the population. The ramification for financial markets being that if investors are linked through a small-world network it will take lesser time for a rumor or a change in a stock recommendation to spread through the network in comparison to a lattice network. Whether the differences in these networks affects the pricing behavior of the system is assessed in Sec. 4.

A random network, which consists of $N$ agents with each agent pair connected with probability $p$, has an abstract nature, and therefore generally serves as a theoretical base line only. However, a special form of a random network, the scale-free network, provides a unique network structure that can affect the dynamics of a system. A popular mechanism for generating a scale-free network is through preferential attachment - a process where an agent chooses their neighbors based on how many neighbors the potential neighbor already has. This process delivers a "the rich get richer" outcome, which results in the degree distribution of the network following a power-law, or at the very least a heavily skewed distribution. This distribution contrasts with the Erdos-Renyi network, where the degree distribution follows a Gaussian distribution. For financial markets this structure would be seen when a large proportion of investors are linked through only a few important investors, for example a Warren Buffet or a rating agency. The implication for the markets and this paper, being that if investors trust these key investors then the volatility of the market can potentially increase as the actions of investors become more aligned as they follow the key investors without considering other information sources.

The first step in generating the lattice network in the implemented model is for the user to set the number of neighbors each investor is to have via the Ring_M parameter. As undirected links are being formed by the investors, the parameter is set as half the number of required neighbors because an investor becomes a neighbor with another investor regardless of whether they create the link or the neighbor creates the link with them. The results for the lattice network were generated with each investor having 4 neighbors, so the parameter is set at 2 . The algorithm places each investor into a list, which is sorted by the investor's numerical identification number. Each investor is then asked to form an undirected link with the next highest investor in the list. The process is repeated for the investor by value of the Ring_M parameter, with the following link being formed with the next highest neighbor. 
The small-world network is formed in a similar manner to the lattice network, with the exception being that based on a given probability an agent will re-wire an existing connection to another agent selected randomly from the population. For low probabilities of re-wiring (5\%) the resulting network will exhibit a high level of clustering, similar to a lattice network, yet a small overall network diameter, as discussed previously. The probability of an agent re-wiring is set at $10 \%$ (prob_of_rewire parameter) for this model. The Erdos-Renyi network is created by providing a probability by which an agent connects to another agent. For this model the this was set at $0.16 \%$ (prob_of_link parameter). Therefore, the average number of neighbors in the population is 4 , with the some agents having up to 13 neighbors, while some agents have no neighbors.

To create a heavily skewed degree distribution for the scale-free network, the user needs to decide on the number of hubs (set by the Ring_M parameter) and the probability (prob_of_link parameter) of an investor connecting to each of those hubs. The algorithm operates with each investor identifying the hubs, which is achieved by the investor searching the population and forming a list with the investors with the highest number of neighbors. The number of investors in the list is determined by the Ring_M parameter. Next, each investor with a probability determined by the prob_of_link parameter links with each of the investors in the list. This process is sufficient to create a scale-free network via a preferential attachment process. This paper uses the following settings: number of hubs (set by the Ring_M parameter $)=10$ and probability of connection (prob_of_link parameter) $=0.20$. Therefore, the average number of neighbors in the investor population equals 4 , which is comparable with the lattice network, noting that the lattice network has a uniform degree distribution while the scale-free is skewed.

In creating the various network topologies, the ability to have the average number of edges and the average number of neighbors consistent across the different network structures was a key consideration. This meant that any difference in the outcome across the networks was not influenced by the number of edges, but solely by the degree distribution of the network, thus relating any findings back to the propositions presented in [30]. The significance of the differing degree distribution is that if an investor only has one neighbor they will have an initial bias toward public and private information, as they collect less opinions, and if they have a lot of neighbors there will be an initial bias towards the information coming from their networks as they collect more opinions. Consideration was given to normalizing the network information but this would have minimized the effect of the different network structures. With investors continually reassessing their trust in each information source, it does not preclude a single neighbor becoming very persuasive. Conversely, an investor with many neighbors may end up having very little trust in them.

To illustrate the different characteristics of the various network topologies, Fig. 2. (all figures in this paper have been produced using [33]) plots the clustering coefficient against the average closeness centrality for each of the networks based on the variables described previously. The key observation is that the scale-free network's 
Network Clustering Coefficient vs. Average Closeness Centrality

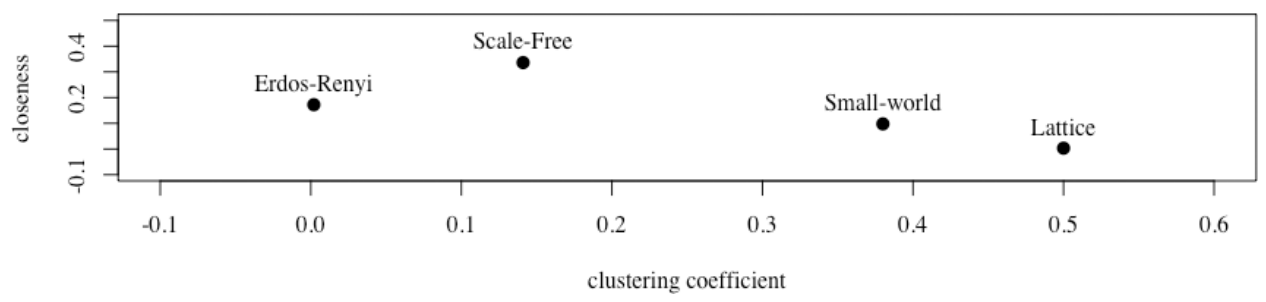

Fig. 2. The clustering coefficient and closeness centrality measures for each of the networks, as per the setting described in Sec. 3.2. The measures are representative of the connectedness of the networks and illustrate the key differences in various topologies.

clustering coefficient is in the middle of the sample, while the lattice and Erdos-Renyi networks are at either end of the spectrum, and its average closeness is the highest. This finding supports the hypothesis that the scale-free network is potentially the most volatile because the opinions of the key investors have sufficient clusters to influence, and their neighbors on average are closer, as indicated by the closeness centrality metric, thereby allowing their influences to spread quickly. Alternatively, while the small-world and lattice networks have a higher clustering coefficient, these clusters may not be large enough nor close enough to form a common herd. Therefore, in these markets the volatility should be lower due to a greater number of competing opinions. The Erdos-Renyi appears to lack both the closeness and clustering of neighbors for a common decision to propagate across the population. Additionally, consistent with the previous description of the network characteristics, while the average number of neighbors in each network is 4 , the degree distribution varies greatly with it being uniform for the lattice network while for the scale-free network it has heavily skewed distribution with a small number of agents linked to many other agents.

\section{Results}

To meet the objective of this paper - to understand if, and how the network topology of investors, and the presence of a dividend affects the behavior of the market, a wide range of parameter settings and experiments were utilized. Firstly, a sensitivity analysis was undertaken to assess the effect on the pricing behavior of the model resulting from varying the initial bias towards listening to one's neighbor $(c 1)$, with $1,2,3$, and 4 used, the network topology and the dividend payout ratio (ratios of: $0,33 \%, 66 \%$, and $100 \%$ were used). The relevance of varying $c 1$ is firstly, to allow the presented model to be verified against original model [13], and whether with similar initial settings (that is no dividend) the model produces the comparable pricing behavior, and secondly to serve as a baseline to compare the results once a dividend is introduced. The parameters chosen for the analysis, which are consistent 
with those used in [13] were: 2,500 investors, 2,500 steps per run, 30 runs per setting, a conviction threshold of 2 , a market depth $(\lambda)$ of 0.25 , a transaction ratio (tr) of 0.02 , and a memory length $(\alpha)$ of 0.95 .

To highlight the differences across the various parameter settings, Fig. 3 provides heat maps that illustrate the average standard deviation in the asset's returns across the 30 runs for each of the different payout ratios, network topologies, and levels of $c 1$. The standard deviation of returns was chosen as that which can capture the volatility of the system. Utilizing the standard deviation in the price, or mean of the price was also assessed, and produced a similar conclusion, which is that the scalefree network produces results which are inconsistent with the other 3 topologies.

From the bottom land hand corners of each of heat maps in Fig. 3, it is evident that the scale-free network has higher levels of volatility for the lower levels of $c 1$, thus distinguishing itself from the other 3 networks. The other 3 networks exhibit similar characteristics in that it is only when $c 1$ is greater than 2 does the volatility increase. Thus, implying that it is the highly skewed degree distribution of the scalenetwork that is responsible for the contrasting outcome. However, in a key finding, the effect of the network topology becomes somewhat redundant once the bias increases. On this basis, and in the interest of maintaining the readability of the paper, from this point onwards, the analysis is restricted to the lattice network, as it allows for a direct comparison with the findings of the original paper [13], and the

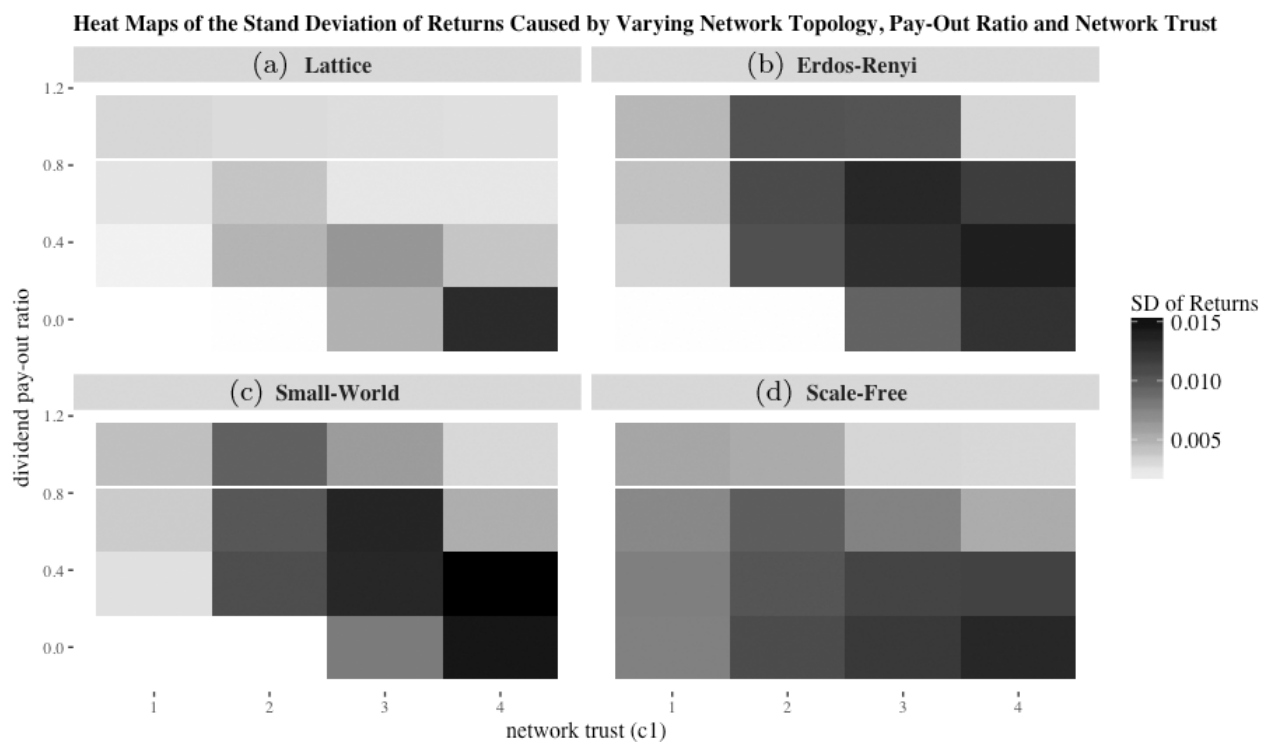

Fig. 3. Heat maps illustrating the sensitivity in the volatility of the risky-asset's returns to the varying network topologies, the dividend payout ratio, and the initial bias that the investors have towards listening to their neighbors. The scale-free network (see Graph (d)) can be differentiated from the other 3 networks based on its higher volatility when there is no dividend paid and when investors have no disposition to following their neighbors, as seen in the bottom left-hand corner of Graph (d). 
scale-free network because it is produces the greatest variation. 2 additional points of interest that are investigated in the remainder of this section, are that as $c 1$ increases the volatility of the systems all increase, and with the exception of the scale-free network, as the payout ratio increases so does the volatility of the system.

Having identified the initial effects of changing the payout ratio, network topology, and the level of network influence, the next step was to analysis the effect of increasing the influence of the public information source $(c 2)$. The intention being to test whether investors having a higher initial faith in the value of public information can prevent the positive feedback loop that results in investors following their neighbors' behaviors, thus ensuring that the risky-asset's price remains in a range closer to its fundamental value. The importance of this step being that the use of public information (the EPS result for the quarter) is a proxy for the fundamental analysis, meaning that if investors have greater trust in this information source, then the asset's price should move in a manner closer to a random walk, as investors receive this information in a random fashion as prescribed by the EMH.

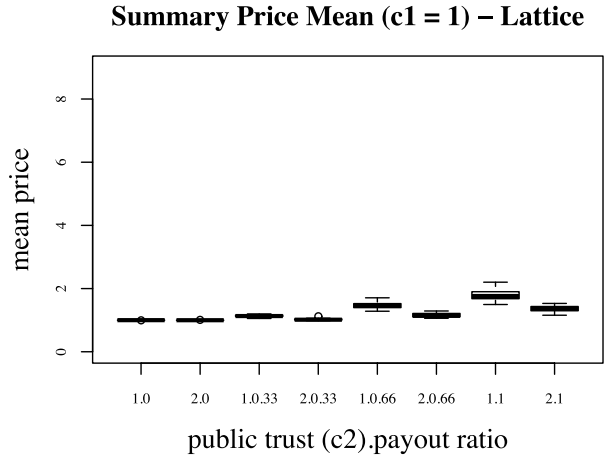

Summary Price Mean $(c 1=1)$ - Scale-Free

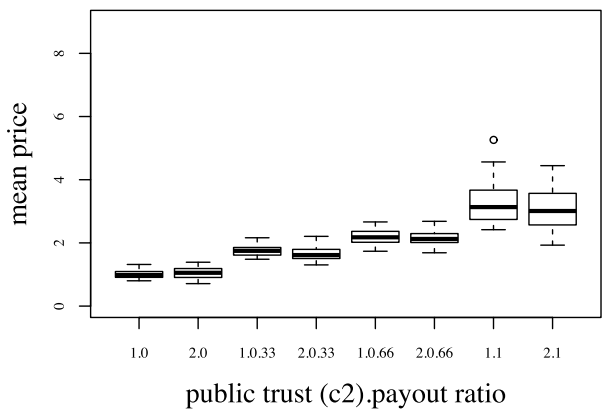

Summary Price Mean $(c 1=4)-$ Lattice

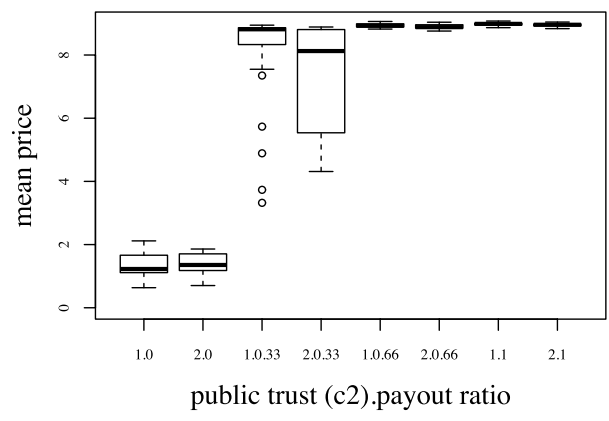

Summary Price Mean $(c 1=4)$ - Scale-Free

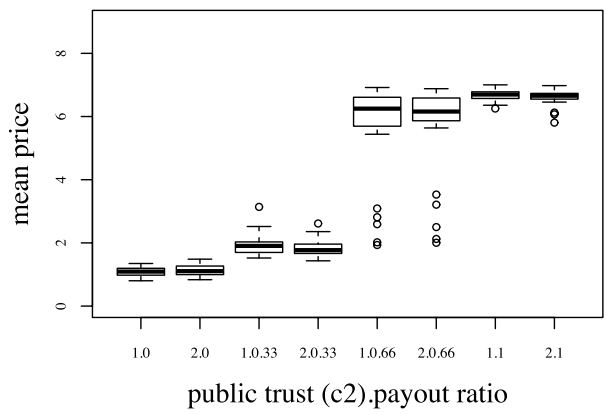

Fig. 4. Boxplots illustrating the mean price of the risky asset over 2,500 for the lattice and scale-free networks with varying payout ratios and public information bias, with the $y$-axis representing the price, with the $x$-axis presenting the various combinations of the initial bias investors have to the public information source, and the chosen payout ratios. 
To gain a greater understanding of variation in the behavior of the asset's price across the various parameters, for the lattice and scale-free networks, Fig. 4 provides boxplots which summarize the behavior of the mean price (the price of the asset across the 2,500 steps) for each of the settings in the parameter sweep. It is evident that there is both a large variation within and between the various networks and the payout ratios. In terms of the lattice network, once the initial bias to listening to your neighbors (the $c 1$ variable) is set at 4 then the mean price is greater than 8 once the dividend payout ratio is increased to $33 \%$ (top right of Fig. 4). For the scale-free network, the $c 1$ variable does not need to be increased to 4 for the mean to move away from 1 (bottom left of Fig. 4). The other point to note is that the scale-free network maintains a higher degree of variability in general across the various settings.

To appreciate why the price differences occur, an analysis of how the time series evolved is required. The movement of the price of the asset utilizing the 2 network topologies, varying levels of $c_{1 i j}$ (as detailed in the heading by the $c 1$ value), and the payout ratio results are illustrated in the fan plots [1] in Figs. 5-8. Fanplots were selected as they effectively illustrate the distribution of the asset's prices resulting from changes to various parameters. By way of explanation, the plots overlay the results of each run with the price plotted against the $y$-axis, with the relevant time

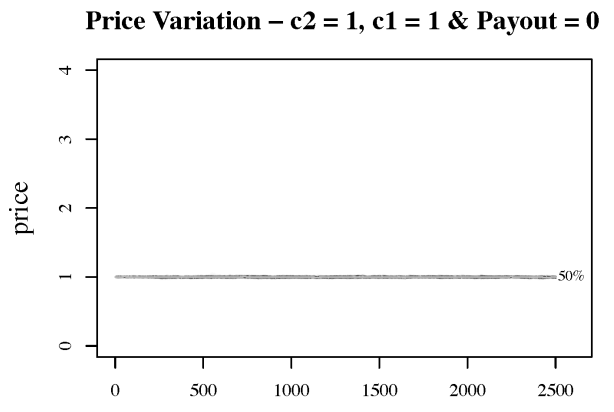

Price Variation $-\mathrm{c2}=1, \mathrm{c1}=1 \&$ Payout $=0.33$

Price Variation $-\mathrm{c2}=1, \mathrm{c1}=1$ \& Payout $=0.66$

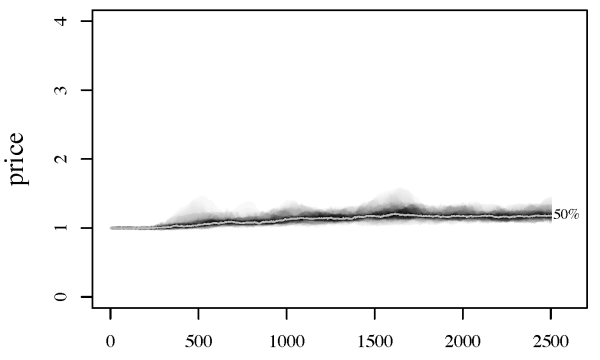

Price Variation $-\mathrm{c2}=1, \mathrm{c} 1=1 \&$ Payout $=1.0$
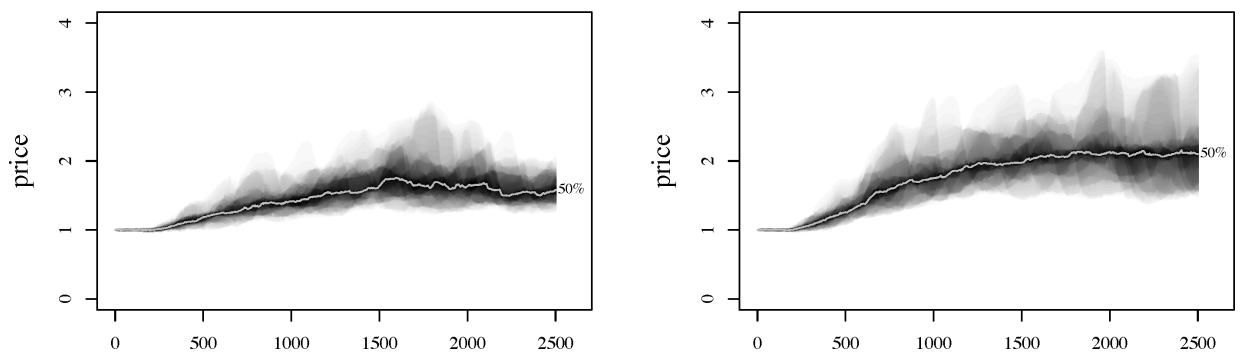

Fig. 5. Fan plots that illustrate the effect, over the length of the simulation run, of varying the dividend payout ratio over time for the lattice network, with the initial bias towards public $(c 2)$ and network information $(c 1)$ equal to 1 . The plots display the density of the price distribution at each step, with a higher density represented by a darker shading. 
Price Variation $-\mathrm{c2}=1, \mathrm{c} 1=4 \&$ Payout $=0$

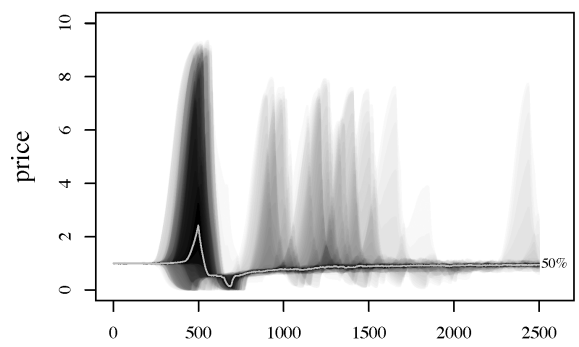

Price Variation $-\mathrm{c2}=1, \mathrm{c1}=4$ \& Payout $=0.66$

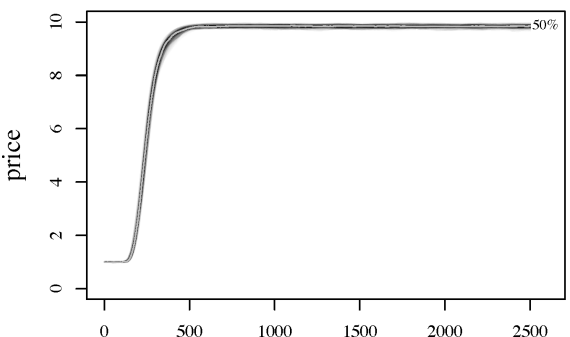

Price Variation $-\mathrm{c2}=1, \mathrm{c1}=4$ \& Payout $=0.33$

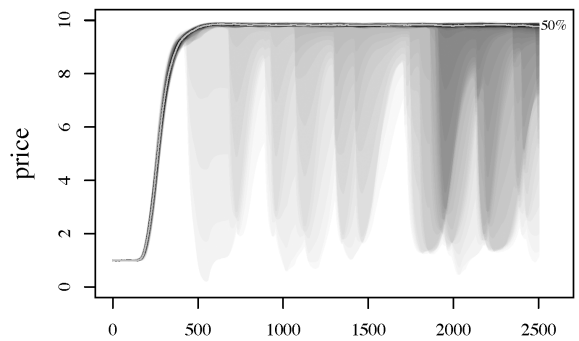

Price Variation $-\mathrm{c} 2=1, \mathrm{c} 1=4 \&$ Payout $=1.0$

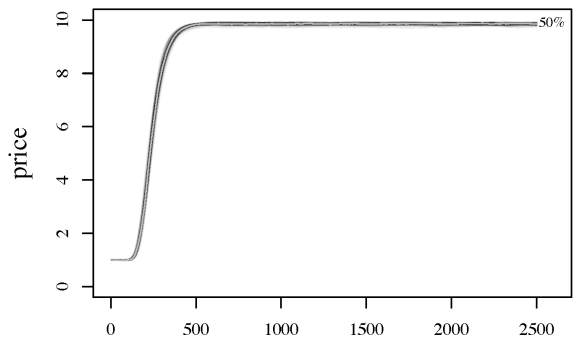

Fig. 6. The effect of varying the payout ratio over time for the lattice network, with the initial bias towards public $(c 2)$ set to 1 and the bias towards network information $(c 1)$ equal to 4 , a setting that is sufficient to have the price move materially from its fundamental value.

Price Variation - Scale-Free with $\mathrm{c} 1=\mathrm{c2}=1$

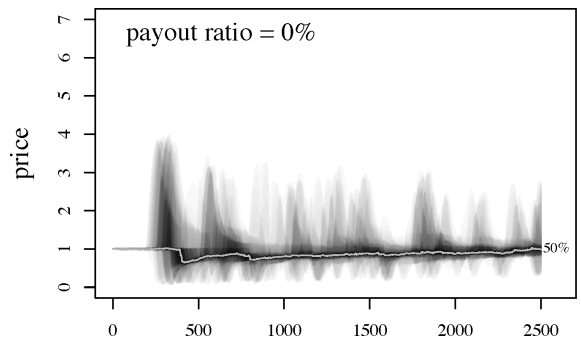

Price Variation - Scale-Free with $\mathrm{c1}=\mathrm{c2}=1$

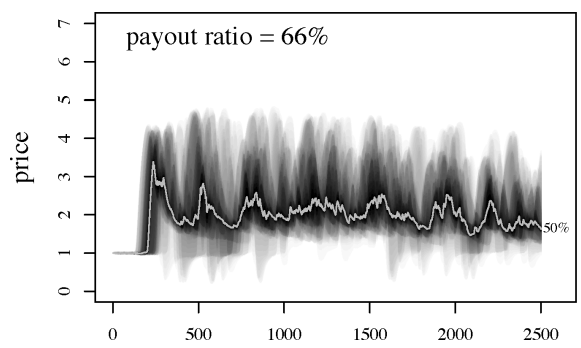

Price Variation - Scale-Free with $\mathrm{c1}=\mathrm{c2}=1$

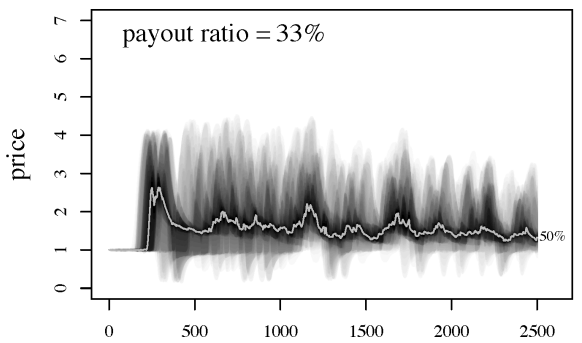

Price Variation - Scale-Free with $\mathrm{c1}=\mathrm{c2}=1$

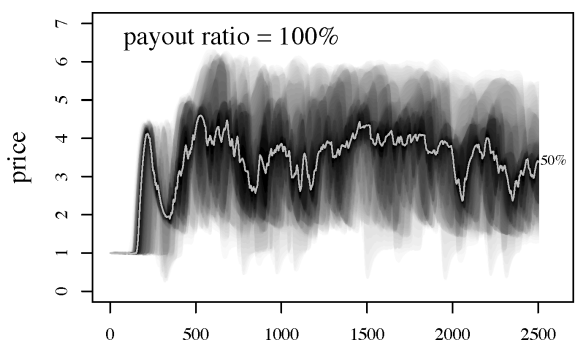

Fig. 7. The relevant fan plots demonstrating the effect of varying the payout ratio over time for the scalefree network, with the initial bias towards public $(c 2)$ and network information $(c 1)$ equal to 1. 
Price Variation - Scale-Free $\mathrm{c2}=1, \mathrm{c1}=4$

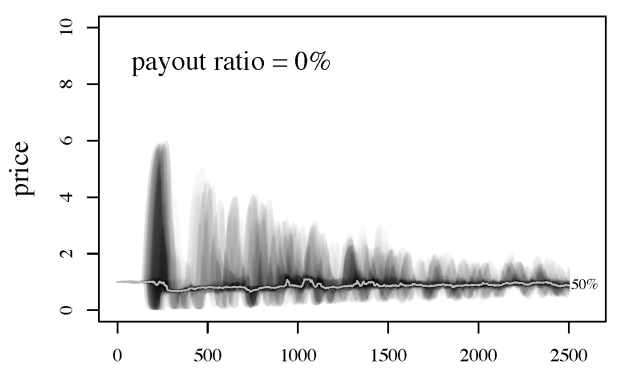

Price Variation - Scale-Free $\mathrm{c2}=1, \mathrm{c1}=4$

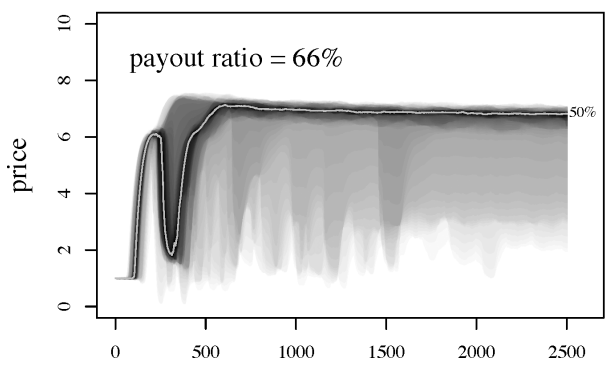

Price Variation - Scale-Free $\mathrm{c2}=1, \mathrm{c} 1=4$

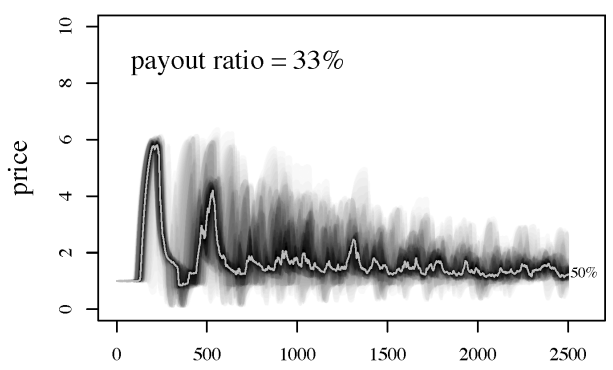

Price Variation - Scale-Free $\mathrm{c2}=1, \mathrm{c} 1=4$

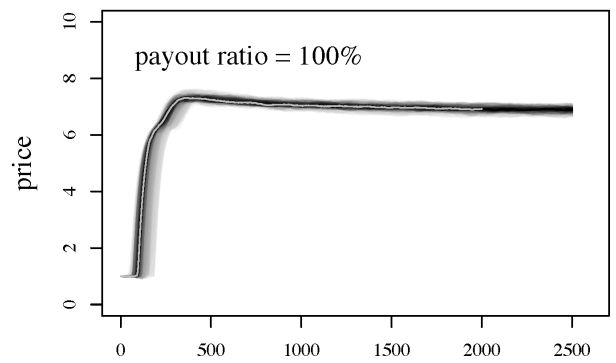

Fig. 8. The relevant fan plots demonstrating the effect of varying the payout ratio over time for the scalefree network, with the initial bias towards public $(c 2)$ set to 1 and the bias towards network information (c1) equal to 4 .

step for the given run plotted on the $x$-axis. This allows the shading to show the density of the distribution, with a darker shading representing a higher density of the price distribution at a given step. The plots were formed from 30 runs for each of the parameter settings noted in the heading. To provide a clear picture in terms of the expected dynamics for each of the scenarios, the median price with the line marked with $50 \%$ is also provided. The lattice network results are seen in Figs. 5 and 6 and the scale-free network results in Figs. 7 and 8.

The upper left-hand corner of Fig. 5 shows the setting of $c 1=c 2=1$ and no dividend. These settings see no volatile price movements with the price series confined to a narrow bound around 1. This result effectively replicates the results from the original model with similar settings thus supporting the use of the extended model. While the price band is narrower than the original model, tests confirmed that the distribution of the returns did not fit a Gaussian distribution. Further verification of the extended model is illustrated in the top left-hand corner of Fig. 6 where the settings are changed to $c 1=4$ with no dividend. Now bubbles, that are comparable in size to the original model [13], appear.

The introduction of a dividend affects the price series in several ways. Firstly, from Fig. 5, where the payout ratio is increased from 0 to 1 , it can be seen the median and the volatility of the price series increase. At this point it is worth remembering 
the key characteristic of the model, namely, that a dividend is only paid when the EPS for a period is greater than 0 and the investors cannot reinvest the proceeds. Also, a sell signal is generated when the EPS result for the asset is lesser than the consensus forecast for the asset, which occurs approximately $50 \%$ of the time. In the instance that EPS $<0$ and the result is below the consensus, the sell signal will not be diluted by the payment of the dividend. However, if the EPS result is positive, a dividend is paid which will boost the returns thus reducing the power of the sell signal, which in turn may limit the growth in trust for the public information. The anticipated effect of this is that the trust that investors generate in the information from their networks is likely to go unchecked by the public information. This in turn will result in the formation of more herds as investors tend to follow the actions of their neighbors rather than act on public information or even their private information. The consequence of the formation of more herds is that the price will increase in range and volatility based on the size of the herd.

From Fig. 5 it was evident that the introduction of a dividend under a regime where there was no initial bias to any information source $(c 1=c 2=1)$ had a mild affect. However, the results in Fig. 6, which illustrate the outcome of setting $c 1$ to 4 (a setting that is responsible for the creation of a bubble [13]), are far more explosive. The most telling result is that once the dividend is introduced the behavior post the inflation of the bubble is very different. In particular, the price of the risky-asset remains materially elevated above its fundamental once the payout ratio is greater than $33 \%$. Even with a payout ratio of $33 \%$, the median price remains in a zone that could be justified as bubble territory (i.e., the median PE ratio is in excess of 140), but the investors experience a high degree of volatility as the boom crashes in some instances, albeit it is not sufficient to move the median price materially from its upper limit.

The significance of these findings is that if there is a high initial bias amongst investors toward listening to your neighbors $(c 1=4)$, the introduction of a dividend sees investors form a buying herd and they can never be persuaded to switch (by analyzing the intentions of the population this process was confirmed). The herding occurs regardless of what the investors' public and private information sources are telling them, including the fact that the EPS of the asset will miss the consensus on average $50 \%$ of the time, thus creating a negative score for public information and providing a sell signal. This phenomenon occurs because the trust the investors place in the actions of their neighbors dominates the decision-making process and the trust does not subside nor can the trust in the other sources build sufficiently to displace it. A more detailed discussion of this point is provided at the end of this section.

To test the null hypothesis that dividends have no effect on price, Kruskal-Wallis rank sum tests were utilized given the nonnormal distribution of the asset's prices. Table 1 reports the mean prices for each of the permutations used in the experiment. The null hypothesis, that for a given level of initial public $(c 2)$ and network trust $(c 1)$ that increasing the payout ratio has no effect, is rejected in all instances - see (a) in Table 1 . The inference being that increasing the payout has a positive effect on the 
Table 1. The mean prices of the risky-asset resulting from the various experimental settings.

\begin{tabular}{|c|c|c|c|c|c|c|c|}
\hline \multirow[b]{2}{*}{ c1 } & \multirow[b]{2}{*}{$\mathrm{c} 2$} & \multicolumn{4}{|c|}{ Dividend payout ratio } & \multirow[b]{2}{*}{ Ave.price } & \multirow[b]{2}{*}{$p$-Value } \\
\hline & & 0.00 & 0.33 & 0.66 & 1.0 & & \\
\hline \multirow[t]{4}{*}{1} & 1 & 1.000 & 1.133 & 1.474 & 1.785 & 1.348 & (a) $<0.01$ \\
\hline & 2 & 0.999 & 1.024 & 1.155 & 1.360 & 1.134 & (a) $<0.01$ \\
\hline & Ave. & 0.999 & 1.078 & 1.315 & 1.573 & 1.241 & \\
\hline & $p$-Value & 0.060 & (b) $<0.01$ & (b) $<0.01$ & (b) $<0.01$ & & \\
\hline \multirow[t]{4}{*}{4} & 1 & 1.350 & 8.111 & 8.937 & 8.979 & 6.844 & (a) $<0.01$ \\
\hline & 2 & 1.393 & 7.287 & 8.892 & 8.953 & 6.631 & (a) $<0.01$ \\
\hline & Ave. & 1.371 & 7.699 & 8.915 & 8.966 & 6.738 & \\
\hline & $p$-Value & 0.204 & (b) 0.013 & (b) 0.012 & (b) 0.035 & & \\
\hline
\end{tabular}

price for an asset despite the asset having a similar earnings profile. To determine the exact relationship and to test its significance, more data points would need to be generated. The table also provides statistical support that in most cases if the initial bias to public information is increased, then the mean price is lower for a given payout ratio - see (b) in Table 1. Therefore, if investors follow fundamental information over following their neighbors then the market will behave in a manner closer to what is prescribed by the EMH.

The results of the utilization of a scale-free network are shown in Figs. 7 and 8, and confirm the initial findings seen in Fig. 3, which is the scale-free network generates results that are significantly different from the lattice network. The key difference, as seen in the top left of Fig. 7. is that a bubble is generated despite there being no dividend, and the investors having no initial bias for listening to their neighbors $(c 1=1)$. A similar result is also generated when the original source of public information is utilized in combination with a scale-free network. This provides another key finding of the extended model, which is if investors are linked in a scalefree network, the market will be more volatile, regardless of the bias that the investors may or may not have towards the information coming from their neighbors.

It has been proposed that the price volatility will be highest in markets with an intermediate level of connectedness yet lower in markets with higher or lower connectedness provides a possible explanation for this result [30]. Indeed, while the scalefree network does have a lower average betweenness measure than the other topologies, it does have intermediate clustering when compared to other network topologies. Importantly, this finding becomes irrelevant once the initial bias to listening to one's neighbors increases, that is the network topology is only important when the investors consider all information sources equally.

Returning to Fig. 7, we can see that as the dividend payout ratio is increased, there are two points of interest. The first is that the median price is greater than 1 in the earlier time periods and the second being that the volatility of the system appears to increase. The median price series also behaves in a contrasting manner to the lattice network, as it appears to revert to 1 (or at least trend down) in all cases except 
the $100 \%$ payout scenario. Interestingly, in the $0 \%$ payout case, the median price drops well below 1 following the initial crash of the bubble, before trending up to 1 . This occurs because the investors continue to sell all the way to the bottom as they remain in a selling herd until the price hits the bottom. The positive payout scenarios still experience the initial crash but the dividend cushions the fall, and the selling herd dissipates earlier in the cycle. Given the contrasting findings for the lattice network, it appears that the effectiveness of a company's management to support their share price via a dividend will be influenced by the network that their investors have formed, thus providing a key insight produced by this paper.

The result of increasing $c 1$ to 4 for the scale-free network, as per Fig. 8, produces a diverse set of findings in comparison to the lattice network. Firstly, the median price only remains elevated once the payout ratio is $66 \%$ or greater. For anything less, the bubble will deflate and the price returns to its fundamental level. Even at the $66 \%$ payout ratio, while the median price remains high, there is far more volatility in the series. The other point is that the bubbles do not reach the same level as the lattice network and there appears a minor downward trend over time, something not seen in the lattice model.

The effect of increasing $c 2$ (the initial bias to public information) provided several interesting insights. From Fig. 4 the initial impression is that while the movement of the median price away from 1 still occurs, it is more gradual and does not reach the same level achieved by $c 1=c 2=1$. A possible explanation, as highlighted in Table 1, is that the effect of higher initial bias to public information is to slow the growth in the trust among neighbors, which in turn diminishes the probability of a herd forming. This occurs because under this regime the influence of the fundamental analysis is not diluted to the same extent in the decision-making process. Therefore this result identifies a mechanism that can prevent the inflation of a bubble, namely, investors having a stronger initial faith in their public information source. The implication is that the use of fundamental information must remain in the population if excessive price movements are to be avoided.

To answer why the different network topologies and payout ratios create such different results, one needs to look at the dynamics regarding the trust that the investors have in the information coming from their neighbors. Noting that bubbles result when the positive feedback mechanism with regard to investors adopting the actions of their neighbors becomes the dominant influence in the decision-making process, Fig. 9 provides boxplots for the average network trust, in the same manner that Fig. 4 did for the prices. What becomes apparent is that the level and deviation of the network trust in each of the scenarios matches the price series, thus providing support to the argument that it is the level of trust investors have in their neighbors that is primarily responsible for driving the market volatility. Of note is the lattice network where $c 1$ is set to 4 . This scenario, which is responsible for an indefinite period of the risky-asset's price remaining well in excess of its fundamental value (when the payout ratio was greater than 33\%), has the highest level of trust and a lower deviation in trust once the payout ratio increases above $33 \%$. In summary from 

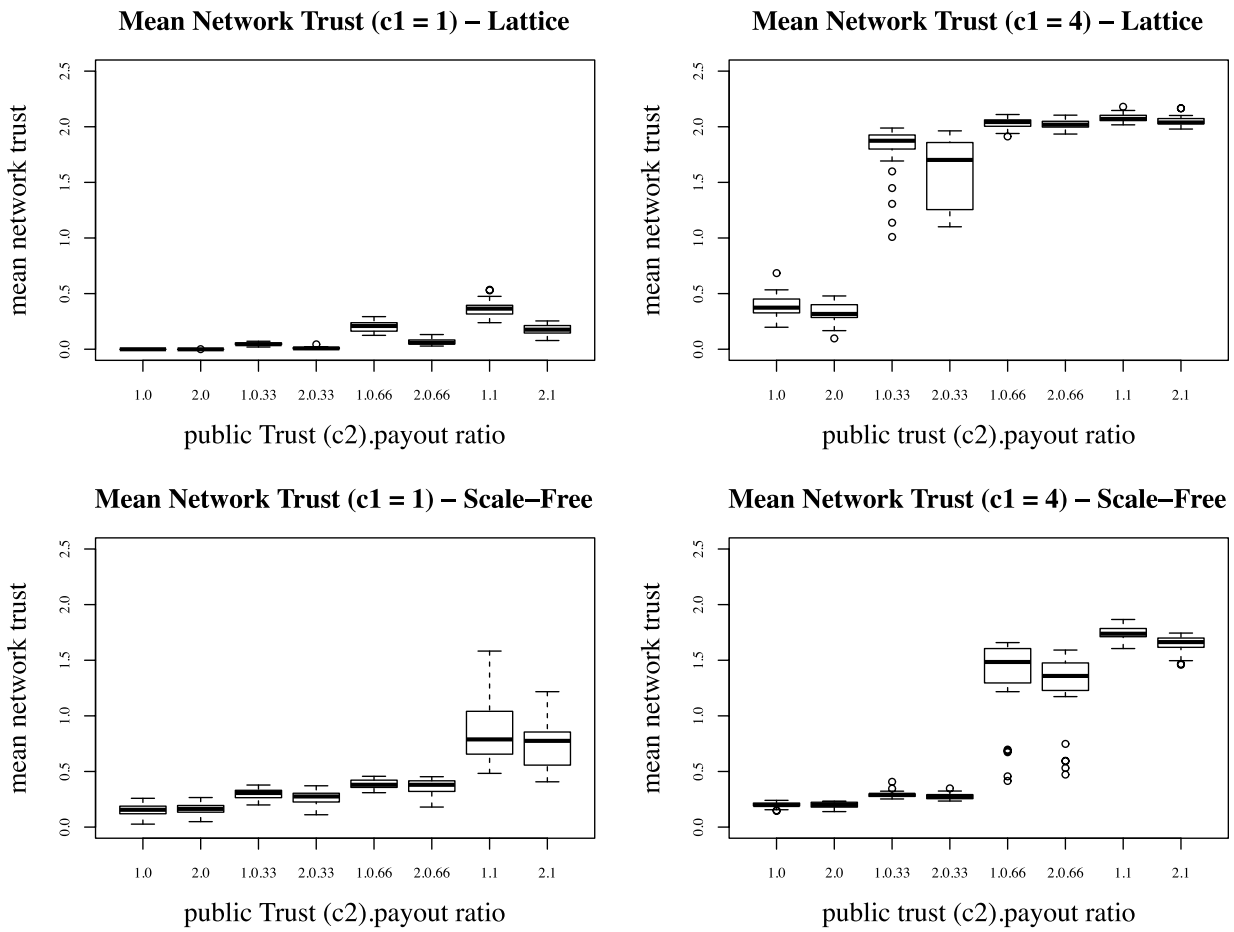

Fig. 9. Boxplots illustrating the mean level of trust that investors have in the information originating from the network. The plots are for the lattice and scale-free networks with varying payout ratios and public information bias, with the $y$-axis representing the level of trust, with the $x$-axis presenting the various combinations of the initial bias investors have to the public information source, and the chosen payout ratios.

Fig. 9, it is apparent that the level of trust can be affected by the network topology that investors form, the dividend that companies pay, or the level of bias investors have to public information.

To gain a greater understanding of how and why the network trust varies under the different levels of initial bias, Figs. 10 and 11 are provided. These fanplots are for the price time series profile of the asset, returns, the "scaling factor" (as defined in Eq. (5) and its utility explained in Sec. 3.1), and the network trust. Looking at Fig. 10, which shows the results from a lattice network with $c 1$ set at 1 , and a $100 \%$ payout ratio, the key observations are that: the price (Graph (a)) grows at a similar rate as the average level of trust that the population has in its neighbor's advice (Graph (d)); the return profile declines over the length of the run (Graph (b)), as percentage price increases become subsequently smaller; and the "scaling factor" accelerates very early in the run (Graph (c)), thus updating the trust at higher rate, before eventually remaining stable. It is important to note that under the scenario of Fig. 10, the agents do not have an initial bias towards listening to their neighbors, so despite the high scaling factor, it takes time for the population to fall into the buying 
Price Variation $-\mathrm{c1}=1 \&$ Payout $=100 \%$

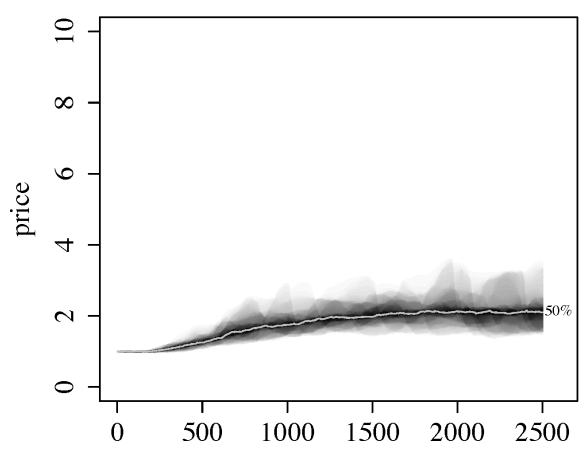

(a)

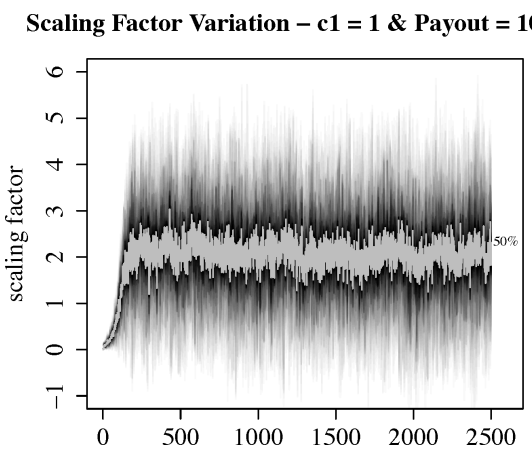

(c)

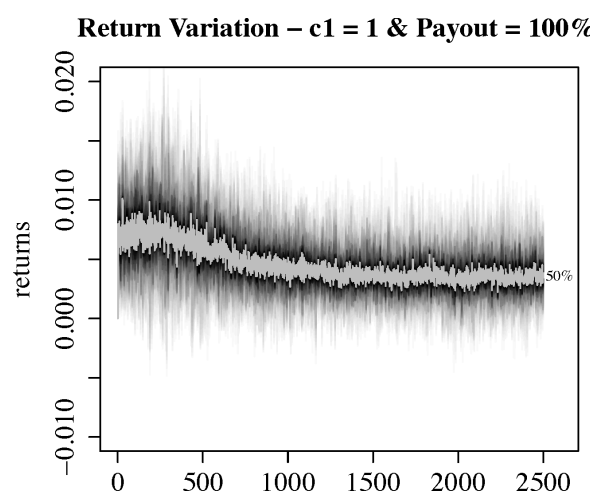

(b)

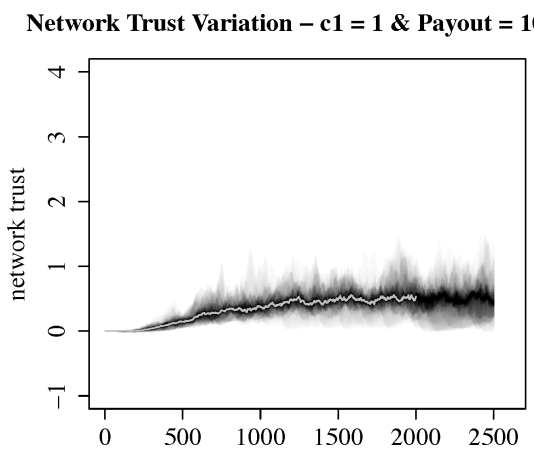

(d)

Fig. 10. The evolution of key variables throughout the simulation with an initial setting of no bias $(c 1=c 2=1)$ to the information sources using the lattice network. Graph (a) shows the price series while an illustration of the associated returns is shown in Graph (b). Graph (c) shows the change in the scaling factor, which is used to update the trust parameters. Graph (d) plots the average network trust across the population, which grows in a similar fashion to the price of the risky asset.

herd because the investors are more balanced in their assessment of the various information sources.

The results seen in Fig. 11, which is from a lattice network topology with $c 1$ set at 4 and a $100 \%$ payout ratio, contrasts significantly to Fig. 10. Apart from the fact that the price rises well in excess of its fundamental value (a price of 1) and never returns, the key finding is that the population takes less time to build trust in the actions of their neighbors, with this resulting in the earlier formation of a material buying herd, resulting in the asset returns being significantly higher in the early periods. In turn, this sees the factor variable peak, and reaches a much higher value in the earlier periods. These observations reveal the process that is responsible for the price of the asset remaining well in excess of its fundamental value indefinitely - a perpetual bubble; that is, it is the rapid growth in trust amongst neighbors as a result of the higher returns due to the combination of a high initial bias to listening to one's 


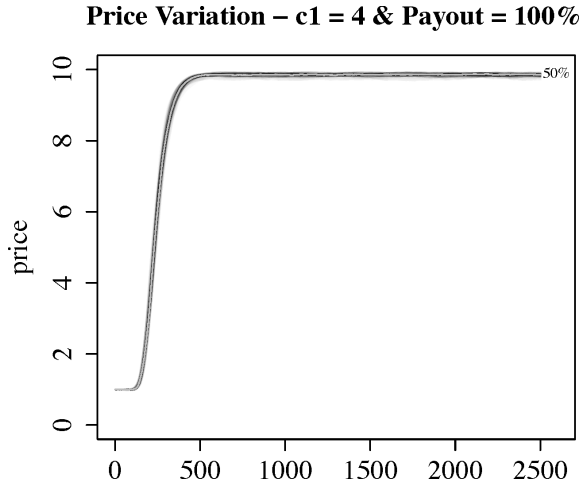

(a)

Scaling Factor Variation $-\mathrm{c} 1=4 \&$ Payout $=100 \%$

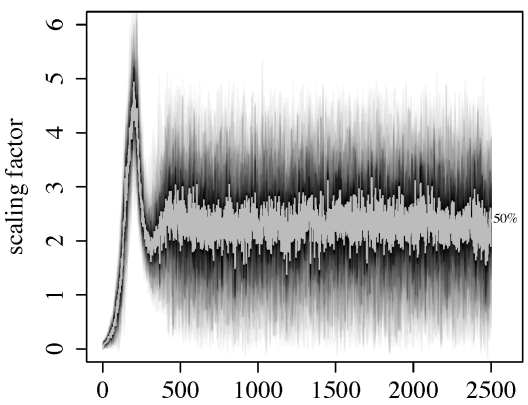

(c)

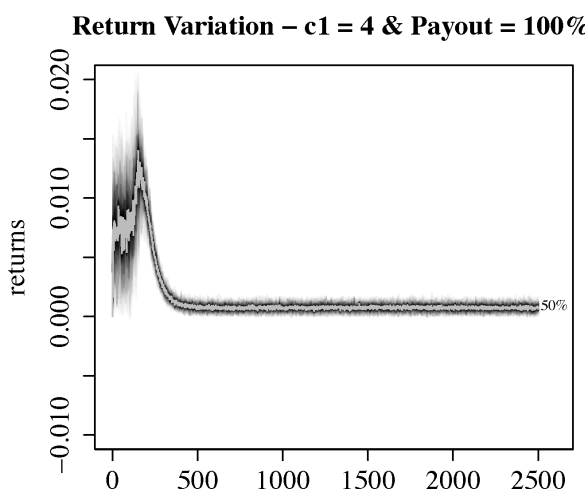

(b)

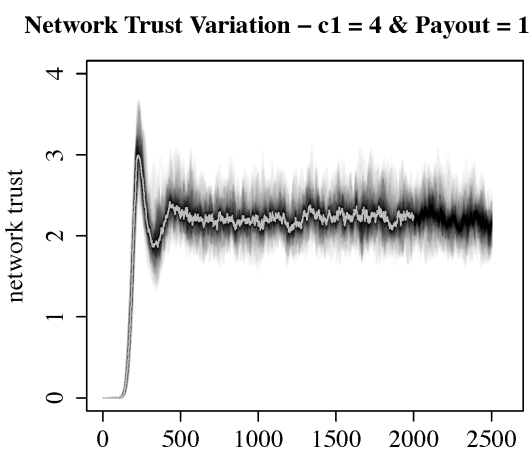

(d)

Fig. 11. The evolution of key variables throughout the simulation with an initial bias to listening to your neighbor $(c 1=4)$ using the lattice network. Graph (a) shows the price of the asset rising, and remaining well in excess of its fundamental value, while Graph (b) shows how the returns evolve. Graph (c) shows that the scaling factor, which is used to update the trust parameters, has an initial peak before settling into a constant range. Graph (d) plots the average network trust across the population, which has a similar early spike in its value.

neighbors, and the introduction of a dividend sees the positive feedback cycle gain sufficient velocity to force the entire population into a buying herd early in the cycle. However, one must remember that it does require an initial bias to listen to your neighbors, and therefore ignoring the fundamental analysis for this dynamic to appear.

Figure 11 does provide evidence that once the initial returns slow the scaling variable and the level of the network trust declines, but not to a level that sees the boom deflate, which would occur once the members of the population left the buying herd. It should also become apparent that the price is in danger of collapsing if the dividend payout ratio is reduced. Parallels to this are evident with the first global financial crisis, as documented in the Extraordinary Popular Delusions and the Madness of Crowds [23]. During the South Sea bubble investors rushed into the market, hoping to replicate the returns of their neighbors and attracted by the 
promise of dividends provided by the likes of the South Sea Company. While there were other contributing factors to implosion of the South Sea bubble, once companies were unable to meet the promised dividend payments the justification for maintaining an investment in them evaporated, and the boom ended in a spectacular crash.

\section{Summary and Conclusion}

The conclusion that can be drawn from this paper is that dividends can make an important contribution to the price of the risky-asset being maintained above its fundamental level as they underwrite returns, which in turn supports the positive feedback process of investors imitating their neighbors. This finding provides evidence that management does have the ability to influence their share prices through their dividend policies, which contrasts with the capital structure irrelevance theory. In reality this may be a short-term view because increasing the payout ratio comes at a cost, namely, a lack of investment in future growth. Therefore, the earnings profile of the company will quite possibly be unsustainable if the dividends remain high. It is this point that provides an obvious extension for this paper, which is to make the payout ratio an endogenous variable within the model and assess the trade-off between future growth and current dividends.

Another implication for a company's management stemming from this paper is that if they are to maximize the positive effect of paying a dividend, they must understand how, and if their shareholders are connected, and whether they have a positive bias to a certain information source. In the instance that a company's shareholders are connected through a few influential investors, as per the scale-free network, then regardless of the actions of management the company's stock price will be more volatile, and, a dividend has limited effect. If investors are more evenly linked, then management has a greater ability to increase the share price of their company by providing a dividend that underwrites the returns for investors. If investors are more inclined to follow their neighbors than the fundamental analysis, then a dividend can help maintain this behavior regardless of the topology of the investor network.

As for the effect of investors being more susceptible to following their neighbors, it was observed by Keynes [16] that markets tended to be more efficient when professional investors, using fundamental analysis, controlled them, and it had been the result of "uneducated" investors, who tended to follow the crowd entering the market, that created the greater volatility. The implication for management from this finding is that if their investors are more inclined to follow the herd then they can expect to see their share prices exhibit greater volatility.

The findings from this paper provide further points of research, namely, adding further dynamics to the model, such as new investors entering the market, how investors attach to their neighbors, and gaining a greater understanding of the networks underlying the real-world investors. All of which support the argument that $\mathrm{ABM}$ and network science still have a considerable contribution to make in understanding the dynamics of financial markets. 


\section{Acknowledgments}

The author would like to thank the anonymous reviewers for providing valuable comments and suggestions to improve the quality of the paper. The publication of this article was funded in part by the George Mason University Libraries Open Access Publishing Fund (OAPF). I would also like to thank George Mason University for providing funding to me through the Presidential Scholarship program, my advisor Prof. Rob Axtell and Prof. Andrew Crooks, for providing insight and guidance in the preparation of this paper.

\section{References}

[1] Abel, G., Fanplot: An R package for visualising sequential distributions, $R J .7(2)$ (2015) $15-23$.

[2] Arthur, W. B., Holland, J. H., LeBaron, B. D., Palmer, R. G. and Tayler, P., Asset pricing under endogenous expectations in an artificial stock market, in The Economy as an Evolving Complex System, eds. Arthur, W. B., Durlauf, S. and Lane D. (Reading, Mass: Addison Wesley, 1997), pp. 15-44.

[3] Bachelier, L., Théorie de la Spéculation (Gauthier-Villars, 1900).

[4] Baker, M. and Wurgler, J., A Catering Theory of Dividends, J. Finance 59(3) (2004) $1125-1165$.

[5] Cont, R., Volatility clustering in financial markets: Empirical facts and agent-based models, in Long Memory in Economics (Springer, 2007), pp. 289-309.

[6] Conway, E., IMF puts total cost of crisis at 7.1 trillion, in The Telegraph (London, 2008), Retrieved from IMF puts total cost of crisis at 7.1 trillion.

[7] Deissenberg, C., van der Hoog, S. and Dawid, H., EURACE: A massively parallel agentbased model of the European economy, Appl. Math. Comput. 204(2) (2008) 541-552.

[8] Fama, E. F., Efficient capital markets: A review of theory and empirical work, J. Finance 25(2) (1970) 383.

[9] Farmer, J. D., Market force, ecology and evolution, Ind. Corp. Change 11(5) (2002) 895-953.

[10] Farmer, J. D., Gallegati, M., Hommes, C., Kirman, A., Ormerod, P., Cincotti, S. and Helbing, D., A complex systems approach to constructing better models for managing financial markets and the economy, Eur. Phys. J. Spec. Top. 214(1) (2012) 295-324.

[11] Gill, A., Biger, N. and Tibrewala, R., Determinants of dividend payout ratios: Evidence from United States, Open Bus. J. 3(1) (2010) 8-14.

[12] Goetzmann, W. N., Bubble investing; Learning from history, Res. Found. Publ. 2016(3) (2016) 149-168.

[13] Harras, G. and Sornette, D., How to grow a bubble: A model of myopic adapting agents, J. Econ. Behav. Organ. 80(1) (2011) 137-152.

[14] Hoffmann, A. O., Jager, W. and V. Eije, J. H., Social simulation of stock markets: Taking it to the next level, J. Artif. Soc. Soc. Simul. 10(2) (2007) 7.

[15] Johnson, N. F., Jefferies, P. and Hui, P. M., Financial Market Complexity (Oxford University Press, Oxford, 2003).

[16] Keynes, J. M., General Theory of Employment, Interest and Money (Atlantic Publishers \& Distributors, 2007).

[17] Kirou, A., Ruszczycki, B., Walser, M. and Johnson, N. F., Computational modeling of collective human behavior: The example of financial markets, in Computational Science ICCS 2008 (Springer Berlin, 2008), pp. 33-41. 
[18] Kothari, S. P., Lewellen, J. and Warner, J. B., Stock returns, aggregate earnings surprises, and behavioral finance, J. Financ. Econ. 79(3) (2006) 537-568.

[19] LeBaron, B., Agent-based computational finance, in Handbook of Computational Economics, Vol. 2 (Elsevier, Amsterdam, 2006), pp. 1187-1227.

[20] LeBaron, B., Agent-based computational finance: Suggested readings and early research, J. Econ. Dyn. Control 24(5) (2000) 679-702.

[21] Li, W. and Lie, E., Dividend changes and catering incentives, J. Financ. Econ. 80(2) (2006) 293-308.

[22] Lux, T., Financial Power Laws: Empirical Evidence, Models, and Mechanism, Economics Working Papers, Christian-Albrechts-University of Kiel, Department of Economics, Kiel (2006).

[23] MacKay, C., Extraordinary Popular Delusions and the Madness of Crowds (Start Publishing LLC, 1841).

[24] MacKenzie, D., An Engine, Not a Camera: How Financial Models Shape Markets (MIT Press, 2008).

[25] Mandelbrot, B. B., The variation of certain speculative prices, J. Bus. 36(4) (1963) 394.

[26] Mandelbrot, B. B. and Hudson, R., The (mis) Behaviour of Markets: A Fractal View of Risk, Ruin and Reward. (Basic Books, 2006).

[27] Miller, M. H. and Modigliani, F., Dividend policy, growth, and the valuation of shares, J. Bus. 34(4) (1961) 411.

[28] Osborne, M. F. M., Brownian motion in the stock market, Oper. Res. 7(2) (1961) 145-173.

[29] Ozsoylev, H. N., Asset pricing implications of social networks, in AFA 2006 Boston meetings paper (Boston, USA, 2006).

[30] Ozsoylev, H. N. and Walden, J., Asset pricing in large information networks, J. Econ. Theory 146(6) (2011) 2252-2280.

[31] Ozsoylev, H. N., Walden, J., Yavuz, M. D. and Bildik, R., Investor networks in the stock market, Rev. Financ. Stud. 27(5) (2014) 1323-1366.

[32] Panchenko, V., Gerasymchuk, S. and Pavlov, O. V., Asset price dynamics with heterogeneous beliefs and local network interactions, J. Econ. Dyn. Control 37(12) (2013) 2623-2642.

[33] R Core Team, R: A Language and Environment for Statistical Computing, R Foundation for Statistical Computing, Vienna, Austria (2013).

[34] Ro, S., Dividends Were Responsible For $42 \%$ of Stock Market Returns Since 1930 , in Business Insider. (2013), Retrieved from www.businessinsider.com stock-returnsprice-dividend-contribution-2013-1.

[35] Schweitzer, F., Fagiolo, G., Sornette, D., Vega-Redondo, F. and White, D., Economic networks: What do we know and what do we need to know? Adv. Complex Syst. 12 (2009) 407-422.

[36] Shiller, R. J. and Pound, J., Survey evidence on diffusion of interest and information among investors, J. Econ. Behav. Organ. 12 (1989) 47-66.

[37] Sornette, D., Physics and Financial Economics (1776-2014): Puzzles, ising and agent-based models, Rep. Prog. Phys. 77(6) (2014) 591-601.

[38] Summers, L. H., Does the stock market rationally reflect fundamental values?, J. Finance 41(3) (1986) 062001.

[39] Watts, D. J. and Strogatz, S. H., Collective dynamics of "Small-World" networks, Nature 393(6684) (1998) 440-442.

[40] Weatherall, J. O., The Physics of Wall Street: A Brief History of Predicting the Unpredictable (Houghton Mifflin Harcourt, 2013).

[41] White, E. N., The fundamental things apply: How to face up to asset market bubbles, Res. Found. Publ. 2016(3) (2016) 169-186. 